\title{
Minimal Representations of Order Types by Geometric Graphs
}

\section{Journal Article}

\section{Author(s):}

Aichholzer, Oswin; Balko, Martin; Hoffmann, Michael (D; Kynčl, Jan; Mulzer, Wolfgang; Parada, Irene; Pilz, Alexander (D); Scheucher, Manfred; Valtr, Pavel; Vogtenhuber, Birgit; Welzl, Emo

Publication date:

2020-12

\section{Permanent link:}

https://doi.org/10.3929/ethz-b-000463986

\section{Rights / license:}

Creative Commons Attribution 4.0 International

\section{Originally published in:}

Journal of Graph Algorithms and Applications 24(4), https://doi.org/10.7155/jgaa.00545 


\title{
Minimal Representations of Order Types by Geometric Graphs
}

\author{
Oswin Aichholzer $\square^{1}$ Martin Balko $\bigsqcup^{2}$ Michael Hoffmann $\bigsqcup^{3}$ \\ Jan Kynčl $\left.\right|^{2}$ Wolfgang Mulzer ${ }^{4}$ Irene Parada $\left.\right|^{5}$ \\ Alexander Pilz $\bigsqcup^{1}$ Manfred Scheucher ${ }^{6}$ Pavel Valtr ${ }^{2}$ \\ Birgit Vogtenhuber ${ }^{1}$ Emo Welzl $\left.\right|^{3}$
}

${ }^{1}$ Institute of Software Technology, Graz University of Technology, Austria

${ }^{2}$ Department of Applied Mathematics, Charles University, Prague, Czech

Republic

${ }^{3}$ Department of Computer Science, ETH Zürich, Switzerland

${ }^{4}$ Institut für Informatik, Freie Universität Berlin, Germany

${ }_{5}^{5}$ Department of Mathematics and Computer Science, TU Eindhoven,

The Netherlands

${ }^{6}$ Institute of Mathematics, Technische Universität Berlin, Germany

\begin{abstract}
In order to have a compact visualization of the order type of a given point set $S$, we are interested in geometric graphs on $S$ with few edges that unambiguously display the order type of $S$. We introduce the concept of exit edges, which prevent the order type from changing under continuous motion of vertices. That is, in the geometric graph on $S$ whose edges are the exit edges, in order to change the order type of $S$, at least one vertex needs to move across an exit edge. Exit edges have a natural dual characterization, which allows us to efficiently compute them and to bound their number.
\end{abstract}

\begin{tabular}{|cccc|}
\hline Submitted: & Reviewed: & Revised: & Reviewed: \\
October 2019 & July 2020 & September 2020 & October 2020 \\
Revised: & Accepted: & Final: & Published: \\
November 2020 & November 2020 & November 2020 & December 2020 \\
Article type: & Communicated by: \\
Regular paper & D. Archambault and C. Tóth \\
\hline
\end{tabular}

E-mail addresses: oaich@ist.tugraz.at (Oswin Aichholzer) balko@kam.mff.cuni.cz (Martin Balko) hoffmann@inf.ethz.ch (Michael Hoffmann) kyncl@kam.mff.cuni.cz (Jan Kynčl) mulzer@inf.fuberlin.de (Wolfgang Mulzer) i.m.de.parada.munoz@tue.nl (Irene Parada) apilz@ist.tugraz.at (Alexander Pilz) scheucher@math.tu-berlin.de (Manfred Scheucher) valtr@kam.mff.cuni.cz (Pavel Valtr) bvogt @ist.tugraz.at (Birgit Vogtenhuber) emo@inf.ethz.ch (Emo Welzl) 


\section{Introduction}

Let $S, T \subset \mathbb{R}^{2}$ be two sets of $n$ labeled points in general position, that is, such that no three points in a set are collinear. We say that $S$ and $T$ have the same order type if there is a bijection $\varphi: S \rightarrow T$ such that any triple $(p, q, r) \in S^{3}$ of three distinct points has the same orientation (clockwise or counterclockwise) as the image $(\varphi(p), \varphi(q), \varphi(r)) \in T^{3}$. The resulting equivalence relation on planar $n$-point sets has a finite number of equivalence classes, the order types [14. Representatives of all the distinct order types of five and six points are illustrated in Figure 1. Among other things, the order type determines which geometric graphs can be drawn on a point set without crossings. Thus, order types appear ubiquitously in the study of extremal problems on geometric graphs.
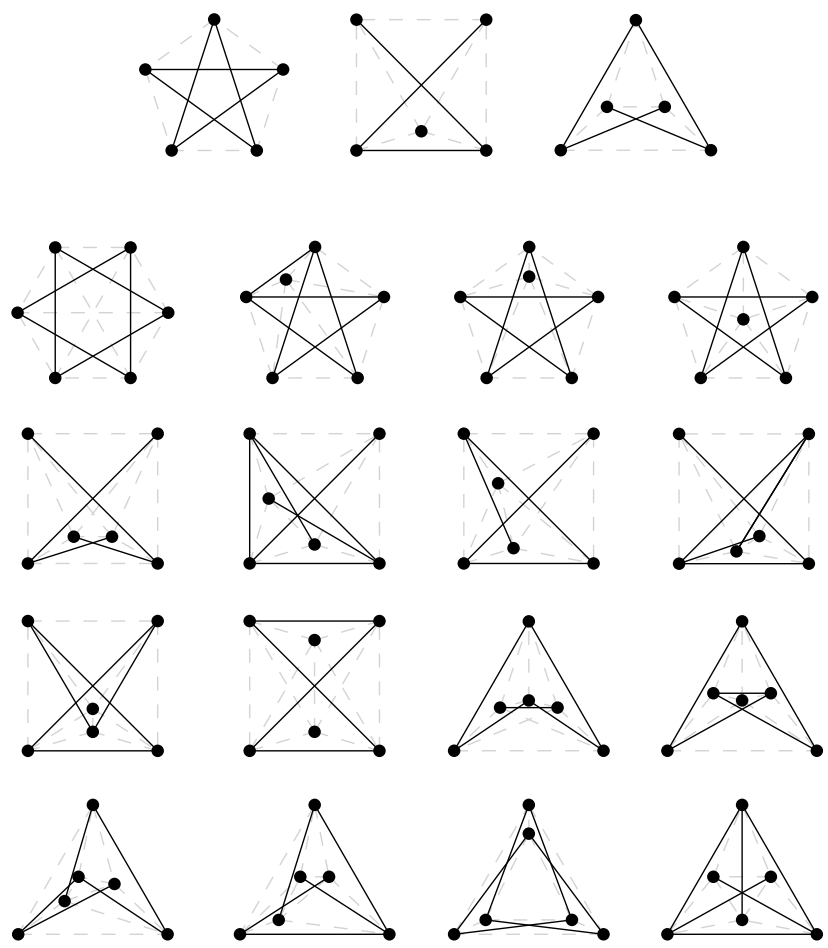

Figure 1: Representatives of the three order types of five points and the sixteen order types of six points in general position. Exit edges are drawn in black.

Now, suppose we have found that an order type is interesting for a problem, and we would like to illustrate it in a publication. One solution is to give explicit coordinates of a representative point set $S$; see Figure 2 (left). This is unlikely to satisfy most readers. We could also present $S$ as a set of dots in a figure. For some point sets (particularly those with extremal properties), the reader may find it difficult to discern the orientation of an almost collinear point triple. To mend this, we could draw all lines spanned by two points in $S$. In fact, it suffices 
to present only the segments between the point pairs (the complete geometric graph on $S$ ). The orientation of a triple can then be obtained by inspecting the corresponding triangle; see Figure 2 (middle). However, such a drawing is rather dense, and we may have trouble following an edge from one endpoint to the other. Therefore, we want to reduce the number of edges in the drawing as much as possible, but so that the order type remains uniquely identifiable. In Figure 2 (right) the triple orientations are unambiguously displayed since continuous deformations that keep the edges straight do not allow to change the orientation of any triple.
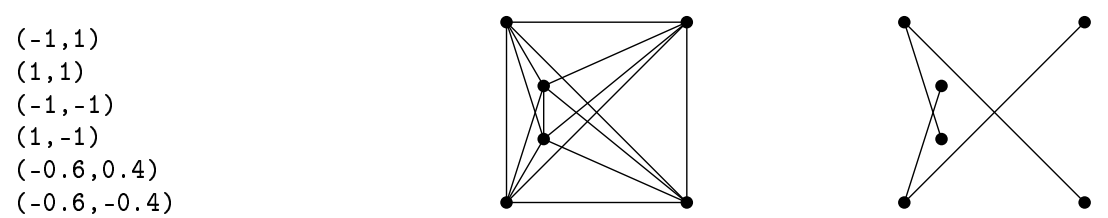

Figure 2: Three different representations of an order type of six points.

Results We introduce the concept of exit edges to capture which edges are sufficient to uniquely identify a given order type in a robust way under continuous motion of vertices. Exit graphs, defined as the geometric graphs whose edges are the exit edges, are supporting for a point set: in an exit graph at least one vertex needs to move across an (exit) edge in order to change the order type. (For precise definitions of these concepts we refer to Definitions 1 and 2.) Though exit edges are defined on a point set, the set of exit edges only depends on the order type and not on the particular representative.

We give an alternative characterization of exit edges in terms of the dual line arrangement, where an exit edge corresponds to one or two empty triangular cells. This allows us to efficiently compute the set of exit edges for a given set of $n$ points in $O\left(n^{2}\right)$ time and space.

Using the more general framework of abstract order types and their dual pseudoline arrangements, we prove that every set of $n \geq 4$ points has at least $(3 n-7) / 5$ exit edges. We also describe a family of $n$ points with $n-3$ exit edges, showing that the best possible lower bound is of order $\Omega(n)$. An upper bound of $n(n-1) / 3$ follows from known results on the number of triangular cells in line arrangements [15]. Thus, compared to the complete geometric graph with $n(n-1) / 2$ edges, using only exit edges saves at least one third of the edges. We present a random construction with a quadratic expected number of exit edges.

Exit graphs are not always minimal supporting graphs. In particular, the requirement of keeping the edges straight together with the non-stretchability of certain pseudoline arrangements can result in exit edges being sometimes unnecessary. The relation between the number of exit edges and the minimum number of edges in a supporting geometric graph is an open question. 
Identification of order types Let $S$ be a set of $n$ labeled points in the plane. A geometric graph on $S$ is a graph with vertex set $S$ whose edges are line segments between their endpoints. A geometric graph is thus a drawing of an abstract graph. Two geometric graphs $G$ and $H$ are isomorphic if there is an orientation-preserving homeomorphism of the plane transforming $G$ into $H$. Each class of this equivalence relation may be described combinatorially by the cyclic orders of the edge segments around vertices and crossings, and by the incidences of vertices, crossings, edge segments, and faces. In the following, we will consider topology-preserving deformations. An ambient isotopy of the Euclidean plane is a continuous map $f: \mathbb{R}^{2} \times[0,1] \rightarrow \mathbb{R}^{2}$ such that $f(\cdot, t)$ is a homeomorphism for every $t \in[0,1]$ and $f(\cdot, 0)=\mathrm{Id}$. Note that if there is an ambient isotopy transforming a geometric graph $G$ into another geometric graph $H$, then no vertex can cross through an edge and $G$ and $H$ are isomorphic. Figure 3 shows an illustration.
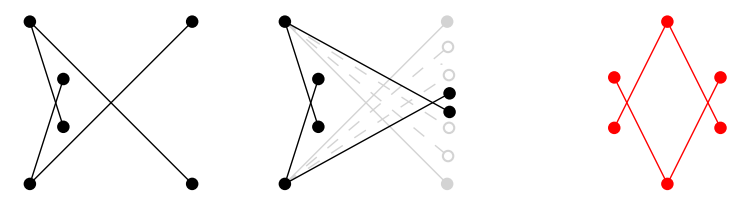

Figure 3: The geometric graph on the left can be transformed by an ambient isotopy into the geometric graph in the middle, but not into the geometric graph on the right.

Definition 1 Let $G$ be a geometric graph on a point set $S$. We say that $G$ is supporting for $S$ if every ambient isotopy $f$ of $\mathbb{R}^{2}$ that, for every $t \in[0,1]$, keeps the images of the edges of $G$ straight (thus, transforming $G$ into another geometric graph) and allows at most one triple of collinear points of $f(S, t)$ also preserves the order type of the vertex set.

Clearly, every complete geometric graph is supporting since all the triangles preserve their orientation, but there are supporting graphs with fewer edges, like the one in Figure 3 (left).

Related work The connection between order types and geometric graphs has been studied intensively, both for planar drawings and for drawings minimizing the number of crossings. For example, it is NP-complete to decide whether a planar graph can be embedded on a given point set [6]. Continuous movements of the vertices of plane geometric graphs have also been considered [2]. The continuous movement of points maintaining the order type was considered by Mnëv [11, 19]. He showed that there are point sets with the same order type such that there is no ambient isotopy between them preserving the order type, settling a conjecture by Ringel [20]. The orientations of triples that have to be fixed to determine the order type are strongly related to the concept of minimal reduced systems 5]. Compact encodings of order types using few bits and allowing for 
fast orientation queries have also been studied. Cardinal et al. [7] presented such an encoding for order types of $n$ points that uses $O\left(n^{2}(\log \log n)^{2} / \log n\right)$ bits, while there are $2^{\Theta(n \log n)}$ order types.

Outline We introduce the concept of exit edges for a given point set. The resulting exit graphs are always supporting, though they are not necessarily minimal. In Section 2 we show that some exit edges are rendered unnecessary by non-stretchability of certain pseudoline arrangements. Despite being nonminimal in general, we argue that exit graphs are good candidates for supporting graphs by discussing their dual representation in pseudoline arrangements (Section 3). This connection allows us to both compute exit edges efficiently and give bounds on their number (Section 4). Supporting graphs in general need not be connected, and two minimal geometric graphs that are supporting for point sets with different order types can be drawings of the same abstract graph; see Figure 1 (right). Thus, the structure of the drawing is crucial. In Section 5 we provide some further properties of the exit graphs. We conjecture that geometric graphs whose edges are the exit edges are not only supporting but also they encode the order type, as discussed in Section 6 .

\section{Exit edges}

To obtain a supporting graph with fewer edges than the complete geometric graph, we select edges so that no vertex of the resulting geometric graph can be continuously deformed (as in Definition 1) to change the order type while preserving isomorphism.

Definition 2 Let $S \subset \mathbb{R}^{2}$ be finite and in general position. Let $a, b, c \in S$ be distinct. Then, $a b$ is an exit edge with witness $c$ if there is no $p \in S$ such that the line $\overline{a p}$ separates $b$ from $c$ or the line $\overline{b p}$ separates a from $c$. We say that ab is an exit edge if there exists a point $c$ such that ab is an exit edge with witness $c$. The geometric graph on $S$ whose edges are all the exit edges is called the exit graph of $S$.

Equivalently, $a b$ is an exit edge with witness $c$ if and only if the double-wedge through $a$ between $b$ and $c$ and the double-wedge through $b$ between $a$ and $c$ contain no point of $S$ in their interior; see Figure 4 (left). We note that the exit graph is invariant under nondegenerate affine transformations.

An exit edge has at most two witnesses. If $|S| \geq 4$ and $a b$ is an exit edge in $S$ with witness $c$, neither $a c$ nor $b c$ can be an exit edge with witness $b$ or $a$, respectively, as otherwise the union of empty regions would cover the rest of the whole plane except the points $a, b$, and $c$. We illustrate the set of exit edges for sets of 5 points in Figure 1 (top).

Exit edges can be characterized via 4-holes. For an integer $k \geq 3$, a (general) $k$-hole in $S$ is a simple polygon $\mathcal{P}$ spanned by $k$ points of $S$ whose interior contains no point of $S$. If $\mathcal{P}$ is convex, we call $\mathcal{P}$ a convex $k$-hole. A point $a \in S$ 

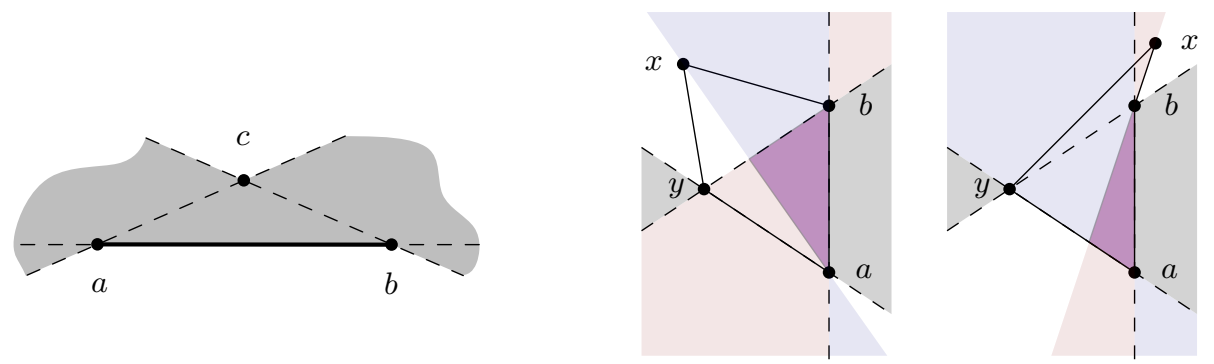

Figure 4: Characterizing exit edges. Left: If the gray region is empty of points, then the edge $a b$ is an exit edge. Right: An illustration of the proof of Proposition 1 .

or an edge $a b$ of the complete geometric graph on $S$ is extremal for $S$ if it lies on the boundary of the convex hull of $S$. A point or an edge that is not extremal in $S$ is internal in $S$.

Proposition 1 Let $S \subset \mathbb{R}^{2}$ be a point set in general position and let $a, b \in S$. Then, $a b$ is not an exit edge of $S$ if and only if the following conditions hold:

1. If $a b$ is extremal for $S$, then $a b$ is an edge of at least one convex 4-hole in $S$.

2. If ab is internal in $S$, then there are two 4-holes abxy and bauv, in counterclockwise order, such that their reflex angles (if any) are incident to ab.

We remark that an internal exit edge either has a witness on both sides or is incident to at least one (not necessarily convex) 4-hole on one side.

Proof: Let $a b$ be an exit edge with a witness $c$ that lies, without loss of generality, to the left of $\overrightarrow{a b}$. Suppose there is a general 4-hole $a b x y$, traced counterclockwise, such that the reflex angle of $a b x y$ (if it exists) is incident to $a b$. We can assume that $y$ lies to the left of $\overrightarrow{a b}$, as in Figure 4 (right). First, suppose that $a b x y$ is convex (this must hold if $a b$ is extremal). Since $a b$ is an exit edge with witness $c$, the line $\overline{a x}$ does not separate $c$ from $b$ and the line $\overline{b y}$ does not separate $c$ from $a$. Thus, $c$ must be inside the 4-hole $a b x y$, which is impossible. Second, suppose that $a b x y$ is not convex (then, $a b$ is internal), and $x$ is to the right of $\overrightarrow{a b}$. Since $a b$ is an exit edge with witness $c$, the line $\overline{b x}$ does not separate $a$ from $c$ and the line $\overline{a y}$ does not separate $b$ from $c$, so $c$ lies inside the 4-hole $a b x y$, again a contradiction.

Conversely, assume that $a b$ is not an exit edge. First, let $a b$ be extremal, and let $p$ be the closest point in $S \backslash\{a, b\}$ to the line $\overline{a b}$. The triangle $a b p$ is a 3-hole in $S$. Since $p$ is not a witness for $a b$, there is a point $q \in S \backslash\{a, b, p\}$ such that, without loss of generality, the line $\overline{b q}$ separates $a$ from $p$. Since $a b$ is extremal, $q$ lies on the same side of $\overrightarrow{a b}$ as $p$ and, in particular, the polygon $a b p q$ is convex. If we choose $q$ so that it is the closest such point to the line $\overline{a p}$, the 
triangles $b p q$ and $a b q$ are 3 -holes in $S$. Altogether, we obtain a convex 4-hole $a b p q$ in $S$.

Second, let $a b$ be internal. Let $p$ be closest in $S \backslash\{a, b\}$ to the line $\overline{a b}$ such that $p$ lies to the left of $\overrightarrow{a b}$. The triangle $a b p$ is a 3 -hole in $S$. Since $p$ is not a witness for $a b$, there is a point $q \in S \backslash\{a, b, p\}$ such that either the line $\overline{b q}$ separates $a$ from $p$ or the line $\overline{a q}$ separates $b$ from $p$. If $q$ lies to the left of $\overrightarrow{a b}$, we obtain a convex 4-hole as in the previous case. Thus, we can assume that all such points $q$ lie to the right of $\overrightarrow{a b}$. We choose the point $q$ so that it is (one of the) closest to the line $\overline{a b}$ among all points that prevent $a b$ from being an exit edge with witness $p$. Without loss of generality, we assume that the line $\overline{b q}$ separates $a$ from $p$. The choice of $q$ guarantees that $b p q$ is a 3-hole in $S$. Thus, $a b q p$ is a 4 -hole in $S$ incident to $a b$ from the left. An analogous argument with a point $p^{\prime}$ from $S \backslash\{a, b\}$ that is closest to $\overline{a b}$ such that $p^{\prime}$ lies to the right of $\overrightarrow{a b}$ shows that there is an appropriate 4-hole in $S$ incident to $a b$ from the right.

Proposition 2 Let $S \subset \mathbb{R}^{2}$ be finite and in general position and, for every $t \in[0,1]$, let $S(t)$ be a continuous deformation of $S$ at time $t$. More formally, let $f: \mathbb{R}^{2} \times[0,1] \rightarrow \mathbb{R}^{2}$ be an ambient isotopy and $S(t)=\{f(s, t) \mid s \in S\}$, for $t \in[0,1]$. Suppose that for every $t \in[0,1]$, there is at most one collinear triple of points in $S(t)$. Let $(a, b, c)$ be the first triple to become collinear, at time $t_{0}>0$. If $c$ lies on the segment $a b$ in $S\left(t_{0}\right)$, then ab is an exit edge of $S(0)$ with witness $c$.

Proof: For $t \in\left[0, t_{0}\right)$, the triple orientations in $S(t)$ remain unchanged, and in $S\left(t_{0}\right)$, the point $c$ lies on $a b$ and the orientations of all triples except $(a, b, c)$ are still unchanged. Thus, for $t \in\left[0, t_{0}\right)$, there is no line through two points of $S(t)$ that strictly separates the relative interior of $a b$ from $c$. In particular, there is no such separating line through $a$ or $b$ in $S(0)$. Hence, $a b$ is an exit edge with witness $c$.

Corollary 1 The exit graph of every point set is supporting.

A line separates $c$ from the relative interior of $a b$ if and only if there is such a separating line through $a$ or $b$. This may suggest that the exit edges are necessary for a supporting graph. However, this is not true in general. For example, in Figure 5 (left), we see a construction by Ringel [20: $a b$ is an exit edge with witness $c$, but $c$ cannot move over $a b$ without violating Pappus' theorem. In this situation, we might consider the abstract order type for the triple orientations we would obtain after moving $c$ over $a b$. Since there is no planar point set with this set of triple orientations, this abstract order type is not realizable. Deciding realizability is (polynomial-time-)equivalent to the existential theory of the reals [19. We will revisit these concepts in Section 4 .

We note that there are point sets where two or more other exit edges prevent a witness $c$ from crossing its corresponding exit edge $a b$; see, for example, Figure 5 (bottom right). Since the two geometric graphs in Figure 5 (right) are not isomorphic, they cannot be transformed into each other by a continuous 

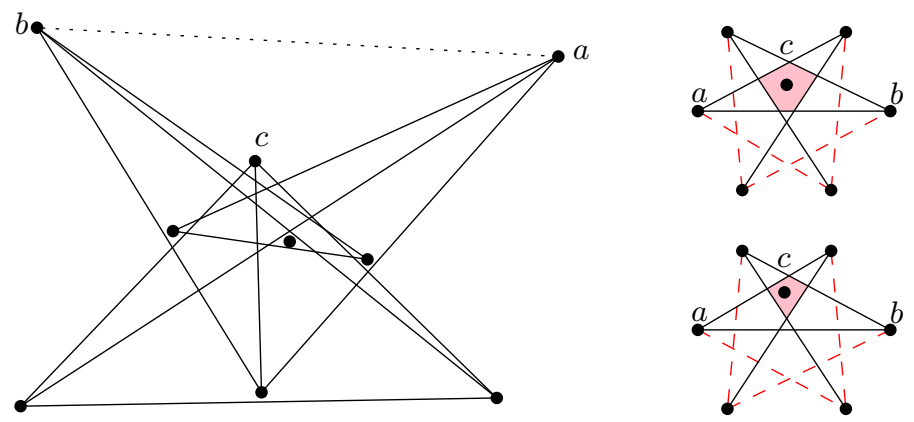

Figure 5: Left: moving $c$ over $a b$ to orient $(a, b, c)$ clockwise, without changing the orientation of other triples, would contradict Pappus's theorem [20]. Right: it is not always possible to move a witness $c$ continuously to the corresponding exit edge $a b$.

deformation as the one used in Definition 1. However, in this example, while $c$ cannot move to $a b$ without changing the order type in Figure 5 (bottom right), if $a b$ were not present, we could first change the point set to the one in Figure 5 (top right) and then move $c$ over $a b$. Thus, $a b$ indeed has to be in a supporting graph.

\section{$3 \quad$ Exit edges and empty triangular cells}

The (real) projective plane $\mathbb{P}^{2}$ is a non-orientable surface obtained by augmenting the Euclidean plane $\mathbb{R}^{2}$ by a line at infinity. This line has one point at infinity for each direction, where all parallel lines with this direction intersect. Thus, in $\mathbb{P}^{2}$, each pair of parallel lines intersects in a unique point.

For a point set $S$ in the Euclidean plane, add a line $\ell_{\infty}$ to obtain the projective plane. We use a duality transformation that maps a point $s$ of $\mathbb{P}^{2}$ to a line $s^{*}$ in $\mathbb{P}^{2}$. In this way, we get a set of lines $S^{*}$ dual to $S$, giving a projective line arrangement $\mathcal{A}$. The removal of a line from $\mathcal{A}$ does not disconnect $\mathbb{P}^{2}$. Since $\mathbb{P}^{2}$ has non-orientable genus 1 , removing any two lines $\ell_{1}$ and $\ell_{2}$ from $\mathbb{P}^{2}$ disconnects it into two components. We call the closure of each of the two components a halfplane 1 determined by $\ell_{1}$ and $\ell_{2}$. The marked cell $c_{\infty}$ is the cell of $\mathcal{A}$ that contains the point $\ell_{\infty}^{*}$ dual to the line $\ell_{\infty}$. By appropriately choosing the duality transformation, we can assume that $\ell_{\infty}^{*}$ lies at vertical infinity. We denote by $w\left(\ell_{1}, \ell_{2}\right)$ the halfplane determined by $\ell_{1}$ and $\ell_{2}$ that does not contain the marked cell.

The combinatorial structure of $\mathcal{A}$, together with the marked cell, determines the order type of $S$. We show how to identify exit edges and their witnesses in dual line arrangements.

We use the marked cell $c_{\infty}$ to orient the lines from $S^{*}$ : first, we orient

\footnotetext{
${ }^{1}$ Here we follow the notation in [15]. In the literature halfplanes are also called wedges.
} 
the lines on the boundary of $c_{\infty}$ in one direction. Then, we iteratively remove lines that have already been oriented, and we define the orientation for the remaining lines from $S^{*}$ by considering the new lines on the boundary of $c_{\infty}$. Then, $c_{\infty}$ is the only cell whose boundary is oriented consistently, that is, it can be traversed completely along the resulting orientation. In particular, for an unmarked triangular cell $\triangle$ in $\mathcal{A}$, the directed edges of $\triangle$ form a transitive order on its vertices, with a unique vertex of $\triangle$ in the middle. We call this vertex the exit vertex of $\triangle$ and the line through the other two vertices of $\triangle$ the witness line of $\triangle$.

Note that if we consider the duality mapping a point $p=\left(p_{x}, p_{y}\right)$ from the real plane to the (non-vertical) line $p^{*}: y=p_{x} x-p_{y}$, then the described orientation procedure corresponds to orienting these dual lines from left to right.

Note that for two points $p, q \in S$ and their dual lines $p^{*}, q^{*} \in S^{*}, w\left(p^{*}, q^{*}\right)$ does not contain the marked cell and therefore its boundary is not oriented consistently.

The next theorem characterizes exit edges and their witnesses in the dual. In its proof we use the following property of projective duality: since it preserves incidences, the condition that no line spanned by two points of $S$ intersects the edge $p q$ is equivalent in $S^{*}$ to $w\left(p^{*}, q^{*}\right)$ not containing any vertex of $\mathcal{A}$.

Theorem 1 Let $S \subset \mathbb{R}^{2}$ be in general position, and let $a, b, c \in S$. Then, $a b$ is an exit edge with witness $c$ if and only if the lines $a^{*}, b^{*}$, and $c^{*}$ bound an unmarked triangular cell $\triangle$ in the arrangement $\mathcal{A}$ of lines from $S^{*}$ so that $c^{*}$ is the witness line of $\triangle$ and the point $\overline{a b}^{*}=a^{*} \cap b^{*}$ is the exit vertex of $\triangle$.

Proof: Let $\triangle$ be the triangular region determined by the intersection of the two halfplanes $w\left(a^{*}, c^{*}\right)$ and $w\left(b^{*}, c^{*}\right)$. By the projective duality, $a b$ is an exit edge with witness $c$ in $S$ if and only if no line of $S^{*}$ intersects $a^{*}$ inside $w\left(b^{*}, c^{*}\right)$ or $b^{*}$ inside $w\left(a^{*}, c^{*}\right)$. In other words, if and only if two sides of $\triangle$, lying on $a^{*}$ and $b^{*}$, contain no intersection with lines from $S^{*}$. This is equivalent to $\triangle$ being a cell of the arrangement $\mathcal{A}$. Moreover, we can recognize $a^{*}$ and $b^{*}$ in $S^{*}$. In the triangular cell $\triangle$ that is the intersection of $w\left(a^{*}, c^{*}\right)$ and $w\left(b^{*}, c^{*}\right)$ the exit vertex is the intersection of $a^{*}$ and $b^{*}$; see Figure 6. Consequently, the exit vertex $a^{*} \cap b^{*}$ is the dual of the line containing the exit edge $a b$ (and vice versa).

Since line arrangements can be efficiently constructed in $O\left(n^{2}\right)$ time [8, 10], Theorem 1 can be used to efficiently compute the set of exit edges.

Corollary 2 Let $S \subset \mathbb{R}^{2}$ be a set of $n$ points in general position. Then the exit edges of $S$ can be enumerated in $O\left(n^{2}\right)$ time by constructing the dual line arrangement of $S$ and checking which cells are unmarked triangular cells.

\section{On the number of exit edges}

Line arrangements can be generalized to so-called pseudoline arrangements. A pseudoline is a closed curve in the projective plane $\mathbb{P}^{2}$ whose removal does not 


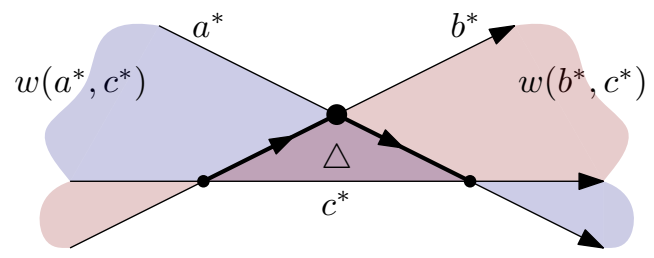

Figure 6: An illustration of the proof of Theorem 1 . If $a b$ is an exit edge with witness $c$ in $S$, then the two bold drawn segments of the corresponding triangular cell are unintersected, and thus, bound an unmarked triangular cell in $S^{*}$. The exit vertex is represented with a black disk.

disconnect $\mathbb{P}^{2}$. A set of pseudolines in $\mathbb{P}^{2}$, where any two pseudolines cross exactly once, determines a (projective) pseudoline arrangement. If no three pseudolines intersect in a common point, the pseudoline arrangement is simple. All notions that we have introduced for line arrangements, such as consistent orientations, exit vertices, or witness lines, naturally extend to pseudolines.

Two pseudoline arrangements are isomorphic if there is an isomorphism of the cell complexes into which they partition $\mathbb{P}^{2}$. A pseudoline arrangement is stretchable if it is isomorphic to a line arrangement, that is, the corresponding cell complexes into which the two arrangements partition $\mathbb{P}^{2}$ are isomorphic. Deciding if a pseudoline arrangement is stretchable is (polynomial-time-)equivalent to the existential theory of the reals [11, 19]. The combinatorial dual analogues of line arrangements and pseudoline arrangements are order types and abstract order types, respectively.

As a consequence of Theorem 1, the maximum number of triangular cells in a simple projective pseudoline arrangement gives an upper bound on the number of exit edges of a point set. However, one triangular cell could be $c_{\infty}$, and there could be pairs of triangular cells with the same exit vertex. We call a configuration of the latter type an hourglass; see Figure 7. We say that the two pseudolines $p$ and $q$ that define the exit vertex of the two triangular cells of an hourglass $H$ slice $H$ and that $H$ is sliced by $p$ and by $q$.
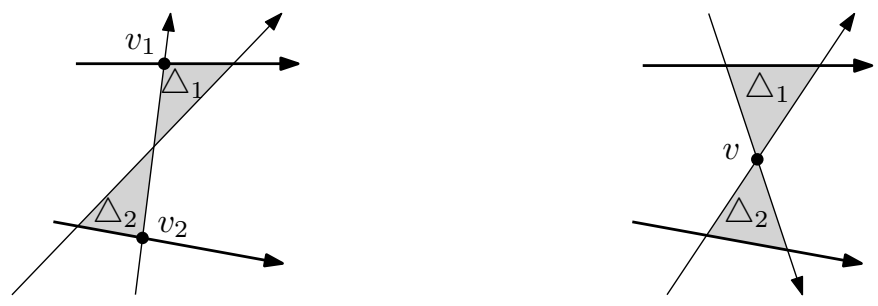

Figure 7: Left: the two triangular cells $\triangle_{1}$ and $\triangle_{2}$ do not form an hourglass, because they share a vertex that is not an exit vertex. Right: the two triangular cells $\triangle_{1}$ and $\triangle_{2}$ form an hourglass because they share an exit vertex. 
Observation 1 A triangular cell can be a part of at most one hourglass.

Observation 2 An exit edge ab with two witness points is dual to an hourglass with exit vertex $\overline{a b}^{*}$.

Any projective arrangement of $n \geq 4$ lines has at least $n$ triangular cells, as each line is incident to at least three triangular cells [17. This is known to be tight. Therefore, taking into account the marked cell $c_{\infty}$ and possible hourglasses, any set of $n \geq 4$ points has at least $\left\lceil\frac{n-1}{2}\right\rceil$ exit edges. We improve this lower bound by bounding from below the difference between the number of triangular cells and the number of hourglasses.

Proposition 3 Any set of $n \geq 4$ points in the plane has at least $(3 n-7) / 5$ exit edges.

For the proof of Proposition 3 we use the following two lemmas. The first is a theorem by Grünbaum [15. Theorem 3.7 on p. 50], and the second can be derived from the proof of that theorem.

Lemma 1 (Grünbaum [15]) In a simple pseudoline arrangement $L$ every pseudoline from $L$ is incident to at least three triangular cells.

Lemma 2 (Grünbaum [15]) Let $L$ be a simple arrangement of pseudolines, and let $H$ be a closed halfplane determined by two pseudolines $\ell_{1}, \ell_{2} \in L$. If two other pseudolines of $L$ cross in the interior of $H$, then there is a triangular cell in $H$ that is incident to $\ell_{1}$ but not to $\ell_{2}$.

Proof of Proposition 3: Let $L$ be a simple projective line arrangement of $n \geq 4$ pseudolines $\ell_{1}, \ell_{2}, \ldots, \ell_{n}$. For each pseudoline $\ell_{i} \in L$, let $t_{i}$ be the number of triangular cells incident to $\ell_{i}$ and $h_{i}$ the number of hourglasses sliced by $\ell_{i}$. Set $x_{i}=t_{i}-h_{i} / 2$. For each pseudoline $\ell_{i} \in L$, there are three possible cases.

Case (i): there is no hourglass sliced by $\ell_{i}$. By Lemma 1 every pseudoline is incident to at least three triangular cells. Thus, we have $x_{i}=t_{i} \geq 3$.

Case (ii): the pseudoline $\ell_{i}$ slices an hourglass together with some pseudoline $\ell_{j}$ and the interior of each of the two halfplanes determined by $\ell_{i}$ and $\ell_{j}$ contains at least one crossing of some other pair of pseudolines. By Lemma 2 , $\ell_{i}$ is incident to the two triangular cells of the hourglass plus at least two other triangular cells, one in each closed halfplane. Thus, $t_{i} \geq 4$. Observation 1 implies $h_{i} \leq t_{i} / 2$. Overall we get $x_{i}=t_{i}-h_{i} / 2 \geq t_{i}-t_{i} / 4 \geq(3 / 4) \cdot 4=3$.

Case (iii): the pseudoline $\ell_{i}$ slices an hourglass together with some pseudoline $\ell_{j}$, and one of the two closed halfplanes $H_{1}$ and $H_{2}$ determined by $\ell_{i}$ and $\ell_{j}$ contains no crossing of any other pair of pseudolines in its interior. Suppose the closed halfplane that contains no further crossing is $H_{1}$. Then, the hourglass sliced by $\ell_{i}$ and $\ell_{j}$ is in $H_{1}$, as the other two lines defining the hourglass do not cross in that halfplane; see Figure 8 (left). Since $H_{1}$ contains no crossing in its interior, it is divided by the other pseudolines into 4 -gons and the two triangular 

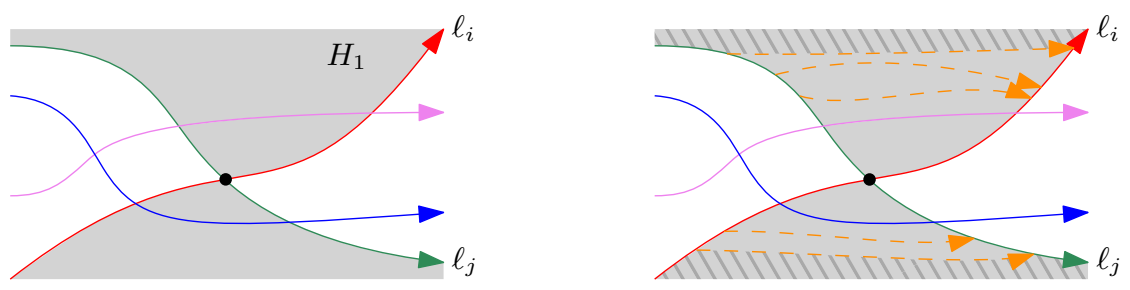

Figure 8: In case (iii), both $\ell_{1}$ and $\ell_{2}$ must bound the marked cell, shown striped on the right picture. Moreover, that cell is bounded by four pseudolines.

cells of the hourglass. In particular, the marked cell is bounded by at most four pseudolines, two of them being $\ell_{i}$ and $\ell_{j}$; see Figure 8 (right). Thus, there can be at most four pseudolines for which case (iii) applies. Notice that in this case $h_{i}=1$, since any other hourglass sliced by $\ell_{i}$ would have one triangular cell in each of the two halfplanes $H_{1}$ and $H_{2}$ and the two triangular cells in $H_{1}$ form the already-counted hourglass (and by Observation 1 they cannot be part of another hourglass). Thus, we can only guarantee that $x_{i} \geq 3-1 / 2=5 / 2$. However, as we showed, this case can happen for at most two pairs of pseudolines.

Let $T$ be the total number of triangular cells in $L$ and let $H$ be the total number of hourglasses. Summing the contributions of cases (i)-(iii), we have

$$
3 T-H=\sum_{i=1}^{n} t_{i}-\frac{1}{2} \sum_{i=1}^{n} h_{i}=\sum_{i=1}^{n} x_{i} \geq 3 \cdot(n-4)+4 \cdot\left(\frac{5}{2}\right)=3 n-2 .
$$

By Observation 1, we have $T \geq 2 H$. Combining these inequalities, we get

$$
T-H=\frac{3 T-H+2(T-2 H)}{5} \geq \frac{3 T-H}{5} \geq \frac{3 n-2}{5} .
$$

By Theorem 1, the number of exit edges in a point set is equal to the number of exit vertices in its dual line arrangement. In general, the number of exit vertices in a pseudoline arrangement is bounded from below by $T-H-1$. Therefore, there are at least $\frac{3}{5} n-\frac{7}{5}$ exit edges.

We do not know if the lower bound in Proposition 3 is tight. The smallest number of exit edges we could achieve is $n-3$ for $n \geq 9$; see Figure 9. We exhaustively checked the set of exit edges for all order types of up to 10 points using the order type database [1] and obtained that this construction with $n-3$ exit edges is optimal for $n=9,10$. Moreover, the order type represented in Figure 9 (left) is the only order type of 9 points that requires 6 exit edges.

The number of triangular cells in a simple arrangement of $n$ lines in the projective plane $\mathbb{P}^{2}$ is at most $n(n-1) / 3[15]$, so there are at most $n^{2} / 3+$ $O(n)$ exit edges. This means that representing an order type with the exit graph instead of the complete geometric graph saves at least one third of the edges. Palásti and Füredi [13] showed that for every value of $n$ there is a simple arrangement of $n$ lines in $\mathbb{P}^{2}$ with $n(n-3) / 3$ triangular cells. Moreover, 

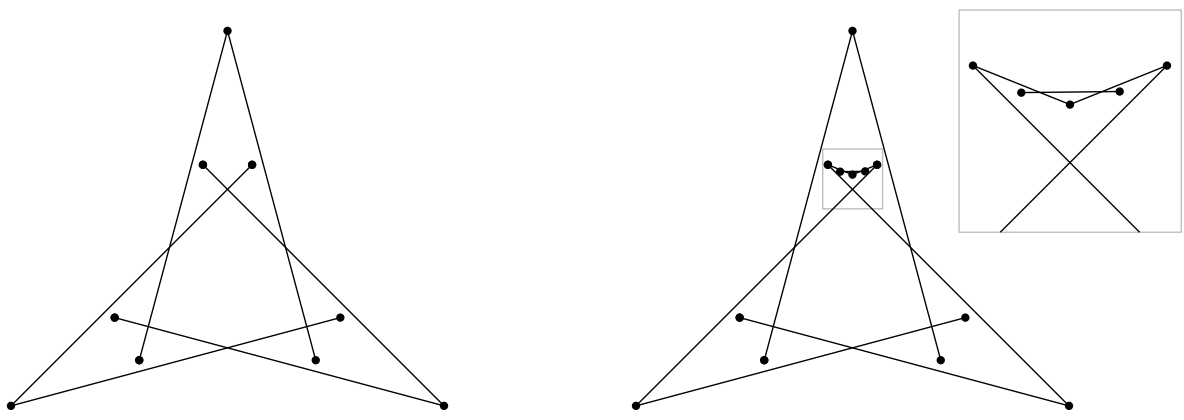

Figure 9: Construction with $n-3$ exit edges.

Roudneff [21] and Harborth [16] proved that the upper bound $n(n-1) / 3$ is tight for infinitely many values of $n$ (see also [4]). The point sets that are dual to the currently-known arrangements that maximize the number of triangular cells have $n^{2} / 6+O(n)$ exit edges, since most of their exit edges have two witnesses. This gives a quadratic lower bound in the worst case, but the leading coefficient remains unknown. It is worth noting that there are line arrangements with no pair of adjacent triangular cells [18, which implies the existence of point sets where every exit edge has precisely one witness.

We now show a random construction with a quadratic expected number of exit edges.

Theorem 2 Let $S=\left\{p_{1}, \ldots, p_{n}\right\}$ be a set of $n$ points in the plane with $p_{i}=$ $\left(i, y_{i}\right)$ for every $i=1, \ldots, n$, where each $y_{i}$ is chosen uniformly at random from the real interval $[1, n]$. Then the expected number of exit edges in $S$ is $\Theta\left(n^{2}\right)$.

The main idea of the proof of Theorem 2 is inspired by the proof of Theorem 2.3 from $[3]$.

Proof: The upper bound $O\left(n^{2}\right)$ on the number of exit edges in $S$ follows from the fact that the number of pairs of points from $S$ is $\left(\begin{array}{l}n \\ 2\end{array}\right)$. In the rest of the proof we establish the lower bound $\Omega\left(n^{2}\right)$.

First, note that all points of $S$ lie in the rectangle $R=[1, n] \times[1, n]$. Assume for convenience that $n$ is divisible by 5 . In the following, we identify each point $p_{i}$ with the number $i$, which is the $x$-coordinate of $p_{i}$. Let $A=\left\{1, \ldots, \frac{n}{5}\right\}$, $B=\left\{\frac{2 n}{5}+1, \ldots, \frac{3 n}{5}\right\}$, and $C=\left\{\frac{4 n}{5}+1, \ldots, n\right\}$. Let $a, b$, and $c$ be fixed integers with $a \in A, b \in B$, and $c \in C$. We now find a lower bound on the probability that $p_{a} p_{c}$ is an exit edge of $S$ with witness $p_{b}$.

The probability that the point $p_{b}$ has vertical distance at most 1 from the line segment $p_{a} p_{c}$ is at least $\frac{1}{n}$, because the points from $\{b\} \times \mathbb{R}$ lying at distance at most 1 from $p_{a} p_{c}$ form a vertical line segment of length 2 , and at least one half of this line segment is contained in $R$.

In the following, we assume that $p_{b}$ has distance at most 1 from $p_{a} p_{c}$. Consider a point $p_{d}$ with $d \in\{a+1, \ldots, n\} \backslash\{b, c\}$. Since $a \in A$ and $b \in B$, we have 


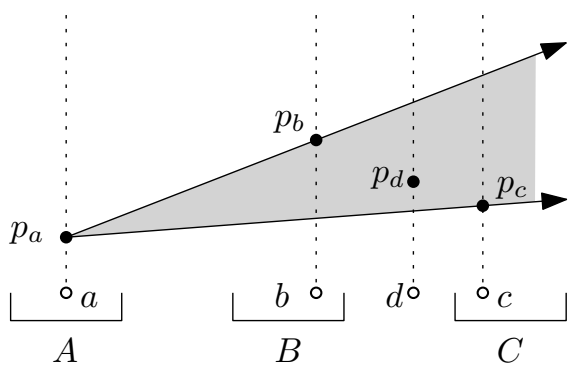

Figure 10: An illustration of the proof of Theorem 2.

$b-a \geq n / 5$ and $d-a \leq n$. Since $p_{b}$ has vertical distance at most 1 from $p_{a} p_{c}$, the vertical side of the triangle $T$ bounded by the vertical line $\{b\} \times \mathbb{R}$ and by the rays $\overrightarrow{p_{a} p_{b}}$ and $\overrightarrow{p_{a} p_{c}}$ has length at most 1 ; see Figure 10 . Since the triangle $T^{\prime}$ bounded by these two rays and by the vertical line $\{d\} \times \mathbb{R}$ is similar to $T$, and since $d-a \leq 5(b-a)$, the vertical side of $T^{\prime}$ has length at most 5 . Thus, the probability that $p_{d}$ lies in the convex wedge spanned by the rays $\overrightarrow{p_{a} p_{b}}$ and $\overrightarrow{p_{a} p_{c}}$ is at most $5 / n$. An analogous argument shows that the probability that a point $p_{d}$ with $d \in\{1, \ldots, c-1\} \backslash\{a, b\}$ lies in the convex wedge spanned by the rays $\overrightarrow{p_{c} p_{a}}$ and $\overrightarrow{p_{c} p_{b}}$ is at most $5 / n$. In total, the probability that $p_{a} p_{c}$ is an exit edge of the point set $\left\{p_{a}, p_{b}, p_{c}, p_{d}\right\}$ with witness $p_{b}$ is at least $1-10 / n$.

Altogether, the probability that $p_{a} p_{c}$ is an exit edge of $S$ with witness $p_{b}$ and that $p_{b}$ is at vertical distance at most 1 from $p_{a} p_{c}$ is at least

$$
\frac{1}{n} \cdot \prod_{d \in\{1, \ldots, n\} \backslash\{a, b, c\}}\left(1-\frac{10}{n}\right)=\frac{1}{n} \cdot\left(1-\frac{10}{n}\right)^{n-3} \geq \frac{1}{n \cdot e^{20}},
$$

where we use the inequality $1-x \geq e^{-2 x}$ for every real $x$ with $0 \leq x \leq 1 / 2$.

Since every exit edge of $S$ has at most two witnesses, the expected number of exit edges of $S$ is at least

$$
\frac{1}{2} \sum_{a \in A} \sum_{b \in B} \sum_{c \in C} \frac{1}{n \cdot e^{20}} \geq \Omega\left(n^{2}\right) .
$$

Combining the point-line duality that maps a point $(a, b)$ to the line $\{(x, y) \in$ $\left.\mathbb{R}^{2}: y=a x-b\right\}$ with Theorem 2 we obtain the following result.

Corollary 3 Let $L=\left\{\ell_{1}, \ldots, \ell_{n}\right\}$ be a set of lines, where $\ell_{i}=\left\{(x, y) \in \mathbb{R}^{2}: y=\right.$ $\left.i \cdot x-b_{i}\right\}$ and where $b_{i}$ is chosen uniformly at random from the real interval $[1, n]$. Then the expected number of triangular cells in the line arrangement induced by $L$ is $\Theta\left(n^{2}\right)$.

\section{Properties of exit graphs}

We present some further results on supporting graphs and exit graphs. 
Theorem 3 Any geometric graph supporting a point set $S \subset \mathbb{R}^{2}$, with $|S| \geq 9$, contains a crossing.

Proof: Let $G$ be a geometric graph with vertex set $S$ without crossings. There is a point set $S^{\prime}$ with a different order type that also admits $G$ : Dujmović [9] showed that every plane graph admits a plane straight-line embedding with at least $\sqrt{n / 2}$ points on a line; as we have a point set with a collinear triple that admits $G$, there are at least two point sets in general position with a different order type that admit $G$. Moreover, one can continuously morph $S$ to $S^{\prime}$ while keeping the corresponding geometric graph planar and isomorphic to $G$ (see, for example, [2]). Therefore, $G$ does not support $S$.

Proposition 4 Let $S$ be a point set in general position in $\mathbb{R}^{2}$ and let $G$ be its exit graph. Every vertex in the unbounded face of $G$ is extremal, that is, it lies on the boundary of the convex hull of $S$.

Note that, as shown in Figure 5 (left), an analogous statement does not hold for general supporting graphs.

Proof: Suppose for contradiction that there is a point $p \in S$ incident to the unbounded face of the exit graph of $S$ and that is internal in $S$, that is, lies in the interior of the convex hull $\operatorname{conv}(S)$ of $S$. This means that there is a polygonal path inside $\operatorname{conv}(S)$ from $p$ to the boundary of $\operatorname{conv}(S)$ such that the interior of this path intersects no exit edge of $S$. Let $\delta(p)$ be the infimum of the lengths of such paths. Since $\operatorname{conv}(S)$ and $S$ are both compact sets, there is a polygonal path $P_{p}$ of length $\delta(p)>0$ from $p$ to the boundary of $\operatorname{conv}(S)$ that has no crossing with exit edges but may pass through other points of $S$. Among all such points $p$, let $r \in S$ be the point for which $\delta(r)$ is the minimum possible. Then $P_{r}$ is a single segment. Let $q$ be the endpoint of $P_{r}$ on the boundary of $\operatorname{conv}(S)$.

If $q$ coincides with an extremal point in $S$, we slightly perturb the point $q$ so that $q$ lies in the interior of an edge of $\operatorname{conv}(S)$ and the line segment $r q$ does not intersect any exit edge of $S$. Let $s$ and $t$ be the endpoints of the edge of $\operatorname{conv}(S)$ containing $q$; see Figure 11 for an illustration.
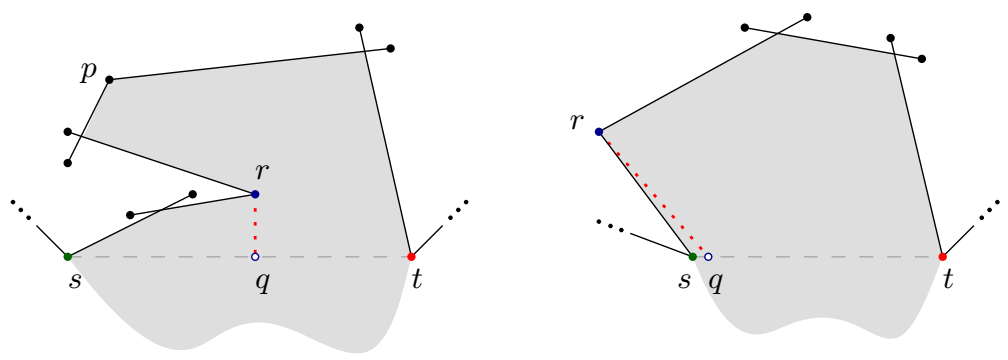

Figure 11: An illustration of the proof of Proposition 4. The path between $r$ and $q$ is drawn as a red dotted line segment. 
Since exit edges are invariant to nondegenerate affine transformations we assume without loss of generality that the following three conditions are satisfied.

(i) The points $r$ and $q$ lie on the $y$-axis, $s$ has negative $x$-coordinate and $t$ has positive $x$-coordinate,

(ii) the point $r$ lies above the line $\overline{s t}$, and

(iii) all points of $S$ have distinct $x$-coordinates.

To obtain a contradiction, we will show that the segment $r q$ intersects the interior of an exit edge of $S$. We will prove this in a dual setting.

By applying the duality transformation mentioned in Section 3 that maps each point $p=\left(p_{x}, p_{y}\right)$ to the (non-vertical) line $p^{*}: y=p_{x} x-p_{y}$, we map the point set $S$ to the dual line arrangement $S^{*}$. Due to the three conditions above, the lines $r^{*}$ and $q^{*}$ are horizontal and the lines $s^{*}$ and $t^{*}$ have a negative and a positive slope, respectively; see Figure 12. By Theorem 1, a triple of points of $S$ representing the endpoints of an exit edge together with its witness, such that the $x$-coordinate of the witness is between the $x$-coordinates of the endpoints of the exit edge, corresponds to a triangular cell in $S^{*}$ where the dual of the witness is the line with median slope bounding this cell.
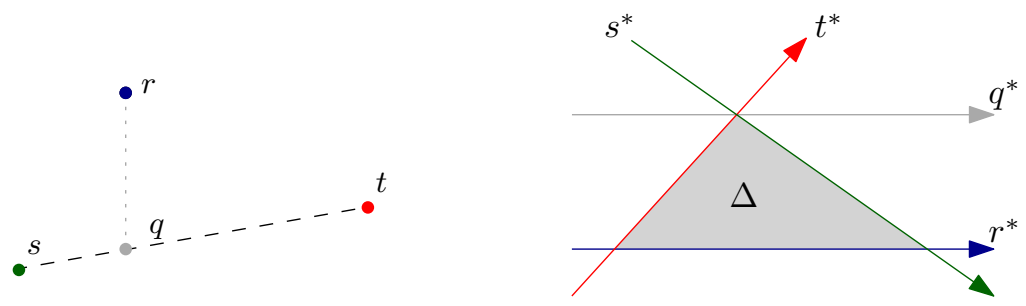

Figure 12: Applying the dual transformation to the point set $S$ (left) and obtaining the line arrangement $S^{*}$ (right).

Let $\triangle$ be the triangular region bounded by the lines $r^{*}, s^{*}$, and $t^{*}$. Since the line segment $s t$ is not an exit edge in $S$, the triangular region $\triangle$ is not a cell in $S^{*}$. Thus, the interior of $\triangle$ is intersected by some line from $S^{*}$. Since $s$ and $t$ are vertices of $\operatorname{conv}(S)$, their duals $s^{*}$ and $t^{*}$ are incident to the upper envelope of $S^{*}$.

Moving a point $p$ vertically down from $r$ to $q$ corresponds to sweeping the dual $S^{*}$ by a horizontal line $p^{*}$ from $r^{*}$ to $q^{*}$. Thus, meeting an exit edge of $S$ with $p$ corresponds to the situation in the dual in which the sweeping line $p^{*}$ meets a vertex of a triangular cell of $S^{*}$ such that the vertex is an intersection of a line with a positive slope and a line with a negative slope. Therefore, the line segment $r q$ crosses an exit edge of $S$ if and only if there is a triangular cell $\triangle^{\prime}$ of $S^{*}$ between $r^{*}$ and $q^{*}$ such that $\triangle^{\prime}$ is bounded by a line with positive slope and a line with negative slope. To obtain a contradiction, we will show that $\triangle$ contains such a triangular cell $\triangle^{\prime}$. 

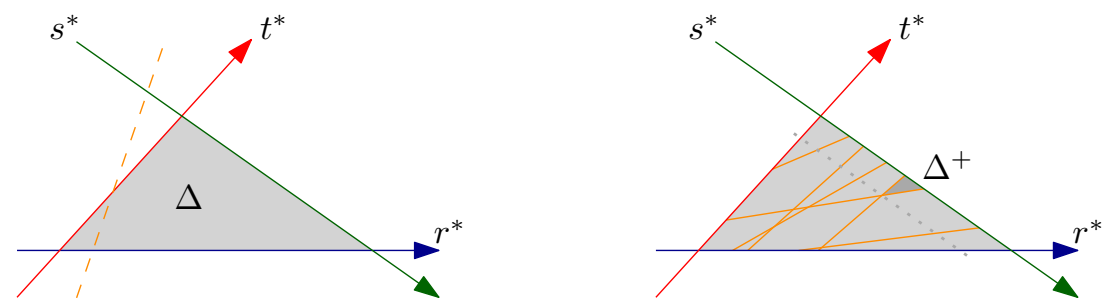

Figure 13: Inserting the set of lines $L^{+}$from $S^{*}$ with positive slope that intersect the interior of $\triangle$. Left: the dashed line cannot be in $L^{+}$since the intersection of $s^{*}$ and $t^{*}$ must be on the upper envelope. Thus, the lines in $L^{+}$ must intersect $s^{*}$ on the boundary of $\triangle$. Right: finding a triangular region $\triangle^{+}$ inside $\triangle$ bounded by $s^{*}$.

We start with the line arrangement containing the lines $r^{*}, s^{*}$, and $t^{*}$. First, we insert the set $L^{+}$of lines from $S^{*}$ with positive slope that intersect the interior of $\triangle$. The goal is to find a triangular region $\Delta^{+}$in $\triangle$ with one edge on $s^{*}$ such that no line from $S^{*}$ with positive slope intersects the interior of $\Delta^{+}$.

Since the lines $s^{*}$ and $t^{*}$ must bound the upper envelope (and are consecutive on it), no line from $S^{*}$ with positive slope can intersect $s^{*}$ above its intersection with $t^{*}$. Thus, the lines from $L^{+}$cannot intersect both $r^{*}$ and $t^{*}$ on the boundary of $\triangle$. By definition, the lines from $L^{+}$must intersect two of the segments bounding $\triangle$ and therefore they must intersect $s^{*}$ on the boundary of $\triangle$; see Figure 13 (left).

Consider the intersection point in $\triangle$ closest to $s^{*}$ produced by two lines $\tilde{r}^{*}$ and $\tilde{t}^{*}$ (that possibly coincide with $r^{*}$ or $t^{*}$ ) from $\left\{r^{*}, t^{*}\right\} \cup L^{+}$. We assume that the slope of $\tilde{t}^{*}$ is larger than the slope of $\tilde{r}^{*}$. Since all the lines from $L^{+}$ intersect $s^{*}$ on the boundary of $\triangle$, the intersection of $\tilde{r}^{*}$ and $\tilde{t}^{*}$ is the leftmost vertex of a triangular cell $\triangle^{+}$(of $\left\{r^{*}, s^{*}, t^{*}\right\} \cup L^{+}$) bounded by $s^{*}$; see Figure 13 (right) for an illustration. Moreover, $\triangle^{+}$is contained in $\triangle$ and it is thus a cell of the arrangement defined by $r^{*}$ and $s^{*}$ together with all the lines with positive slope from $S^{*}$ (including $t^{*}$ and all the lines in $L^{+}$).

We now consider the lines from $S^{*}$ with negative slope. We denote by $L^{-}$ the set of lines from $S^{*}$ with negative slope that intersect the interior of $\Delta^{+}$. Analogously as before, we show that there is a triangular cell $\triangle^{\prime}$ of $S^{*}$ inside $\triangle^{+}$ with one edge on $\tilde{t}^{*}$.

Since the lines $s^{*}$ and $t^{*}$ must bound the upper envelope, lines from $S^{*}$ with negative slope and steeper than $s^{*}$ must intersect $s^{*}$ above its intersection with $t^{*}$ (and therefore above its intersection with $\tilde{t}^{*}$ ). Thus, the lines from $L^{-}$ cannot intersect both $\tilde{r}^{*}$ and $s^{*}$ on the boundary of $\triangle^{+}$; see Figure 14 (left). By definition, the lines from $L^{-}$must intersect two of the segments bounding $\triangle^{+}$ and therefore they must intersect $\tilde{t}^{*}$ on the boundary of $\Delta^{+}$.

In an analogous manner as before, the intersection in $\triangle^{+}$closest to $\tilde{t}^{*}$ defines a triangular cell $\triangle^{\prime}$ inside $\triangle^{+}$bounded by $\tilde{t}^{*}$; see Figure 14 (right). Thus, we found a triangular cell $\triangle^{\prime}$ of $S^{*}$ contained in $\triangle$ bounded by a line with positive 

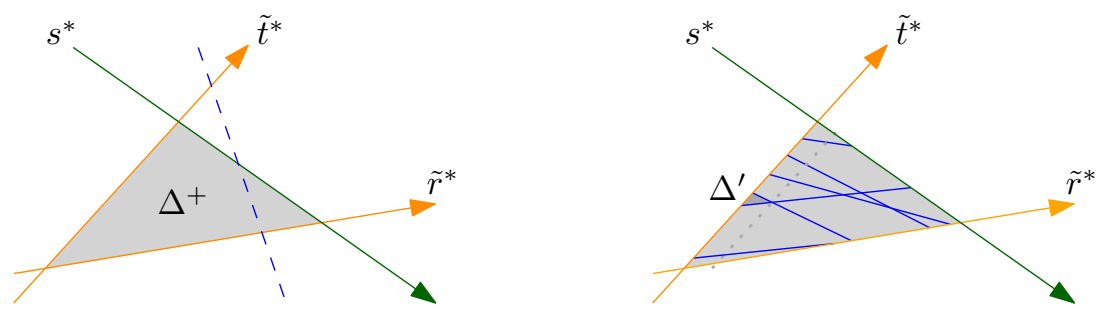

Figure 14: Inserting the set of lines $L^{-}$from $S^{*}$ with negative slope that intersect the interior of $\Delta^{+}$. Left: the dashed line cannot be in $L^{-}$since the intersection of $s^{*}$ and $\tilde{t}^{*}$ must be on the upper envelope. Thus, the lines in $L^{-}$must intersect $\tilde{t}^{*}$ on the boundary of $\triangle^{+}$. Right: finding a triangular cell $\triangle^{\prime}$ inside $\triangle^{+}$bounded by $\tilde{t}^{*}$.

slope and a line with negative slope. Altogether, by duality, this implies that the segment $r q$ crosses an exit edge of $S$, which is a contradiction.

\section{Concluding remarks}

We conjecture that the geometric graph $G$ of exit edges not only is supporting for $S$, but also that any point set $S^{\prime}$ that is the vertex set of a geometric graph isomorphic to $G$ has the same order type as $S$. One might conjecture that already knowing all exit edges and their witnesses (in the dual line arrangement, all triangular cells and their orientations) is sufficient to determine the order type. Surprisingly, this turns out to be false.

A counterexample is sketched in Figure 15 as a dual (stretchable) pseudoline arrangement of 14 lines in the projective plane, based on an example by Felsner and Weil [12. It consists of two arrangements of six lines in the Euclidean plane that are combinatorially different, but share the set of triangular cells and their orientations. While the exit edges and their witnesses are the same for the two different order types, the corresponding exit graphs are not isomorphic.

In the dual of that example the order of the triangular cells along each pseudoline differs, but that extra information is not enough to distinguish the two order types: We can modify the pseudoline arrangements in Figure 15 by, essentially, duplicating pseudolines 1-6 and making a pseudoline and its duplicate cross between the crossings with two red pseudolines (7-14). In Figure 16 we present an illustration. It shows two pseudoline arrangements with the same triangular cells (including their orientations) and the same order of triangular cells along each pseudoline. However, the corresponding order types are not the same (see for example the number of extremal points). Note that the dual point sets of the pseudoline arrangements in Figure 16 can be obtained from the ones in Figure 15 by adding a copy of points 1-6 close to the original respective points. Thus, we cannot reconstruct the order type from that information. 

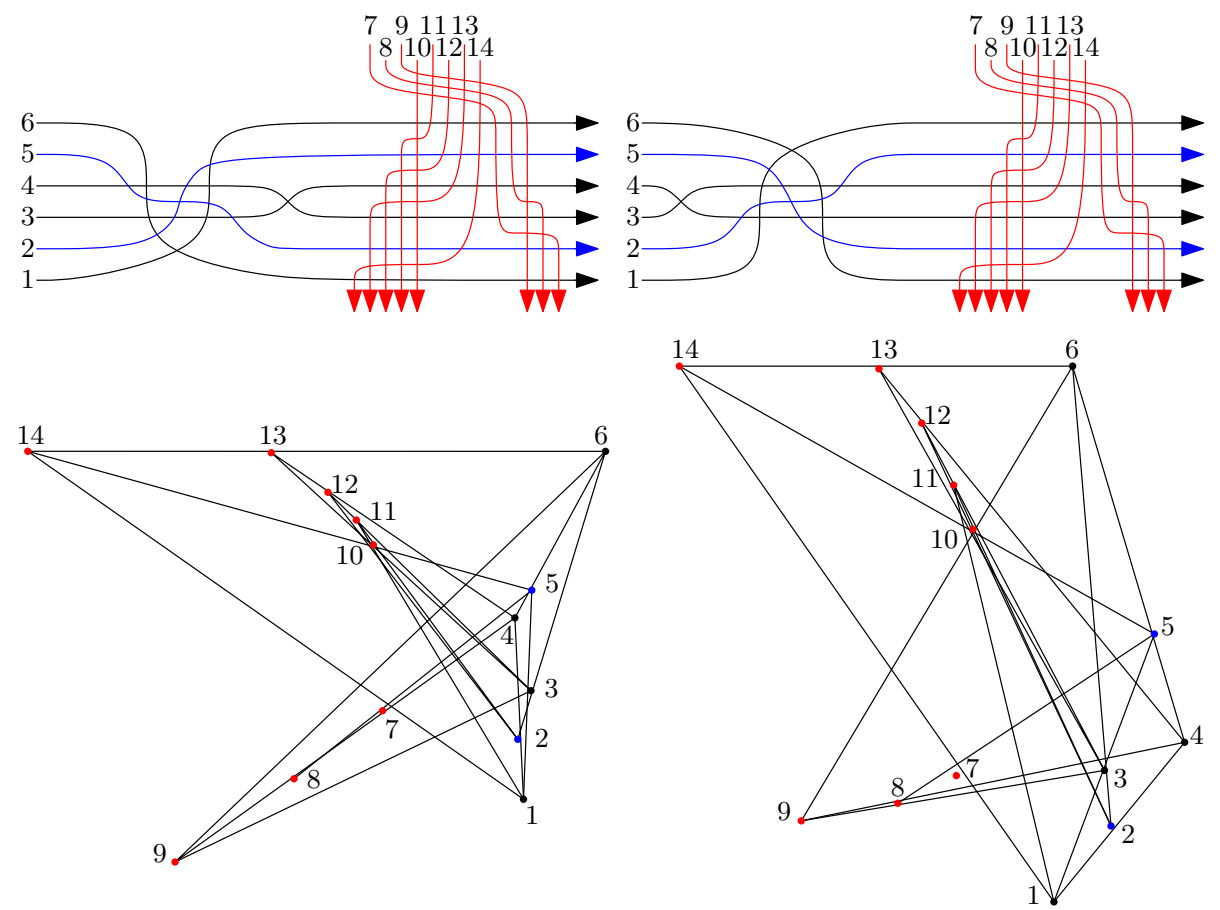

Figure 15: Top: two arrangements of 14 pseudolines with the same set of triangular cells (extending [12, Figure 3]). No triangular cell is crossed by the line at infinity. Bottom: corresponding dual point sets and exit graphs. The order types are not the same (see for example the number of extremal points).

\section{Acknowledgments}

This work was initiated during the Workshop on Sidedness Queries, October 2015, in Ratsch, Austria. We thank Thomas Hackl, Vincent Kusters, and Pedro Ramos for valuable discussions.

This research is supported by the German Science Foundation (DFG), the Austrian Science Fund (FWF), and the Swiss National Science Foundation (SNSF) within the collaborative DACH project Arrangements and Drawings. O.A., I.P., and B.V. were supported by Austrian Science Fund (FWF) grant W1230 and I 3340-N35. M.B., J.K., and P.V. were supported by grant no. 1819158S of the Czech Science Foundation (GAČR). M.B. and J.K. were supported by Charles University project UNCE/SCI/004. M.B. has received funding from European Research Council (ERC) under the European Union's Horizon 2020 research. M.H. and E.W. were supported by SNSF Project 200021E-171681. A.P. was supported by a Schrödinger fellowship of the Austrian Science Fund (FWF): J-3847-N35. M.S. was partially supported by DFG Grant FE 340/12-1. W.M. was partially supported by ERC StG 757609 and DFG Grant 3501/3-1. 

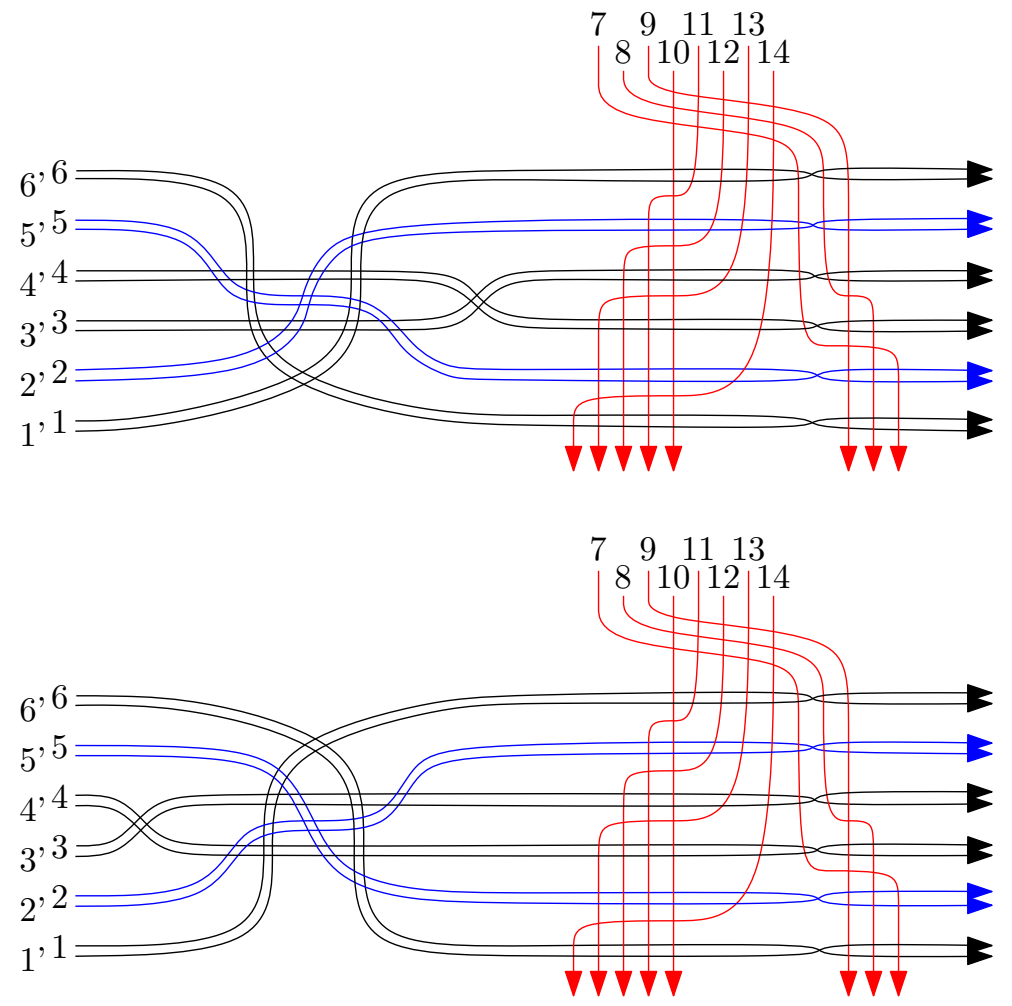

Figure 16: Two arrangements of 20 pseudolines with the same set of triangular cells (extending [12, Figure 3]) and with the same ordering of the triangular cells along the pseudolines, but corresponding to different order types. 
JGAA, 24(4) 551-572 (2020)

\section{References}

[1] O. Aichholzer. The order type database. Last accessed: Nov. 12, 2020. URL: http://www.ist.tugraz.at/aichholzer/research/rp/ triangulations/ordertypes/.

[2] S. Alamdari, P. Angelini, F. Barrera-Cruz, T. M. Chan, G. Da Lozzo, G. Di Battista, F. Frati, P. Haxell, A. Lubiw, M. Patrignani, V. Roselli, S. Singla, and B. T. Wilkinson. How to morph planar graph drawings. SIAM J. Comput., 46(2):824-852, 2017. doi:10.1137/16M1069171.

[3] I. Bárány and Z. Füredi. Empty simplices in Euclidean space. Can. Math. Bull., 30(4):436-445, 1987. doi:10.4153/CMB-1987-064-1.

[4] J. Blanc. The best polynomial bounds for the number of triangles in a simple arrangement of $n$ pseudo-lines. In Geombinatorics, volume 21, pages 5-17, 2011. URL: https://edoc.unibas .ch/47402.

[5] J. Bokowski and B. Sturmfels. On the coordinatization of oriented matroids. Discrete Comput. Geom., 1:293-306, 1986. doi:10.1007/BF02187702.

[6] S. Cabello. Planar embeddability of the vertices of a graph using a fixed point set is NP-hard. J. Graph Algorithms Appl., 10(2):353-363, 2006. doi:10.7155/jgaa.00132.

[7] J. Cardinal, T. M. Chan, J. Iacono, S. Langerman, and A. Ooms. Subquadratic encodings for point configurations. J. Comput. Geom., 10(2):99126, 2019. doi:10.20382/jocg.v10i2a6.

[8] B. Chazelle, L. J. Guibas, and D.-T. Lee. The power of geometric duality. BIT, (25):76-90, 1985. doi:10.1007/BF01934990.

[9] V. Dujmović. The utility of untangling. J. Graph Algorithms Appl., 21(1):121-134, 2017. doi:10.7155/jgaa.00407.

[10] H. Edelsbrunner, J. O'Rourke, and R. Seidel. Constructing arrangements of lines and hyperplanes with applications. SIAM J. Comput., 15(2):341-363, 1986. doi:10.1137/0215024.

[11] S. Felsner and J. E. Goodman. Pseudoline arrangements. In C. D. Tóth, J. O'Rourke, and J. E. Goodman, editors, Handbook of Discrete and Computational Geometry, pages 125-157. CRC Press, 3rd edition, 2017. doi:10.1201/9781315119601.

[12] S. Felsner and H. Weil. A theorem on higher Bruhat orders. Discrete Comput. Geom., 23(1):121-127, 2000. doi:10.1007/PL00009485.

[13] Z. Füredi and I. Palásti. Arrangements of lines with a large number of triangles. Proc. Am. Math. Soc., 92(4):561-566, 1984. doi:10.2307/2045427. 
572 O. Aichholzer et al. Minimal Representations of Order Types

[14] J. E. Goodman and R. Pollack. Multidimensional sorting. SIAM J. Comput., 12(3):484-507, 1983. doi:10.1137/0212032.

[15] B. Grünbaum. Arrangements and spreads. AMS, 1972. URL: https: //bookstore.ams.org/cbms-10/.

[16] H. Harborth. Some simple arrangements of pseudolines with a maximum number of triangles. Ann. N. Y. Acad. Sci., 440(1):31-33, 1985. doi: $10.1111 / \mathrm{j} .1749-6632.1985 . \mathrm{tb} 14536 . \mathrm{x}$.

[17] F. Levi. Die Teilung der projektiven Ebene durch Gerade oder Pseudogerade. Ber. Math.-Phys. Kl. Sächs. Akad. Wiss. Leipzig, 78:256-267, 1926. In German.

[18] D. Ljubić, J.-P. Roudneff, and B. Sturmfels. Arrangements of lines and pseudolines without adjacent triangles. J. Comb. Theory. Ser. A, 50(1):2432, 1989. doi:10.1016/0097-3165(89) 90003-4.

[19] N. E. Mnëv. The universality theorems on the classification problem of configuration varieties and convex polytope varieties. In Topology and Geometry - Rohlin Seminar, volume 1346 of Lecture Notes in Math., pages 527-544. Springer, 1988. doi:10.1007/BFb0082792.

[20] G. Ringel. Teilungen der Ebene durch Geraden oder topologische Geraden. Math. Z., 64:79-102, 1956. In German. doi:10.1007/BF01166556.

[21] J.-P. Roudneff. On the number of triangles in simple arrangements of pseudolines in the real projective plane. Discrete Math., 60:243-251, 1986. doi:10.1016/0012-365X(86)90016-6. 